\title{
High Index SMES Device for Gravitomagnetic Field Generation
}

\author{
Gary V. Stephenson (1) \\ Seculine Consulting, Cupertino, CA, USA \\ Email: seculine@gmail.com
}

How to cite this paper: Stephenson, G.V. (2021) High Index SMES Device for Gravitomagnetic Field Generation. Journal of High Energy Physics, Gravitation and Cosmology, 7, 367-376. https://doi.org/10.4236/jhepgc.2021.72020

Received: December 23, 2020

Accepted: March 28, 2021

Published: March 31, 2021

Copyright $\odot 2021$ by author(s) and Scientific Research Publishing Inc. This work is licensed under the Creative Commons Attribution International License (CC BY 4.0).

http://creativecommons.org/licenses/by/4.0/

\section{(c) (i) Open Access}

\begin{abstract}
A method is described for creating a measurable unbalanced gravitational acceleration using a gravitomagnetic field surrounding a superconducting toroid as described by Forward (1962). An experimental Superconducting Magnetic Energy Storage (SMES) toroid configuration of wound superconducting nanowire is proposed to create a measurable acceleration field along the axis of symmetry, providing experimental confirmation of the additive nature of a Lense-Thirring derived gravitomagnetic field. In the present paper, gravitational coupling enhancement of this effect is explored using a high index or high permittivity material, as predicted by Sarfatti (2020) using his modification to Einstein's General Relativity Field Equations for gravitational coupling in matter.
\end{abstract}

\section{Keywords}

Gravitational, Gravitomagnetic, Lense-Thirring, Superconducting Magnetic Energy Storage, SMES, Nanorods, Nanowires, Super Dielectric Materials, SDM, Super Capacitors, High Permittivity, Gravitomagnetic Permeability, Gravitational Coupling

\section{Introduction}

When Forward [1] first proposed a gravitomagnetic toroid for unbalanced gravitational force production in 1962 that any experimental realization was quite impractical. However, recent advances in high temperature superconducting (HTSC) nanorod wire (nanowire) technology, described recently by Stephenson et al. [2] and Rieken et al. [3], have enabled a new class of superconducting magnetic energy storage (SMES) devices operating at current densities sufficient to develop measurable gravitomagnetic fields.

In the present paper, an experimental SMES toroid configuration is pro- 
posed that uses a super dielectric material (e.g. high permittivity super capacitor) to substantially improve gravitational coupling of mass flow to the curvature of spacetime as predicted by Sarfatti [4]. Depending on the nature of gravitational coupling, it is predicted that a set of standard accelerometers could measure acceleration fields along the axis of symmetry of the toroidal coil, thus providing experimental confirmation of the additive nature of the gravitomagnetic fields, as well as the production of a linear component of the overall acceleration field, see Figure 1 for details.

In the instantiation of Forward's gravitational generation coil described in this paper, superconducting electron flow provides the change in mass current in the toroid. A high permittivity material such as a super dielectric material (SDM) [5] is added to the space in the center of the toroid to improve gravitational coupling. Alternatively or additionally high permittivity insulators may be used as an exterior jacket to the conducting portion of the nanowire so as to further boost gravitational coupling, as gravitomagnetic effects are largest adjacent to the mass flow.

\section{Gravitomagnetic Force Equation for Toroid Mass Flow}

As first developed in Forward 1962 Ref. [1] the linear force $G_{f}$ developed by gravitomagnetic force in the mass flow toroid of Figure 1 is given by Equation (1):

$$
G_{f}=\left(\frac{\eta}{4 \pi}\right)\left(\frac{N \dot{T} r^{2}}{R^{2}}\right)
$$

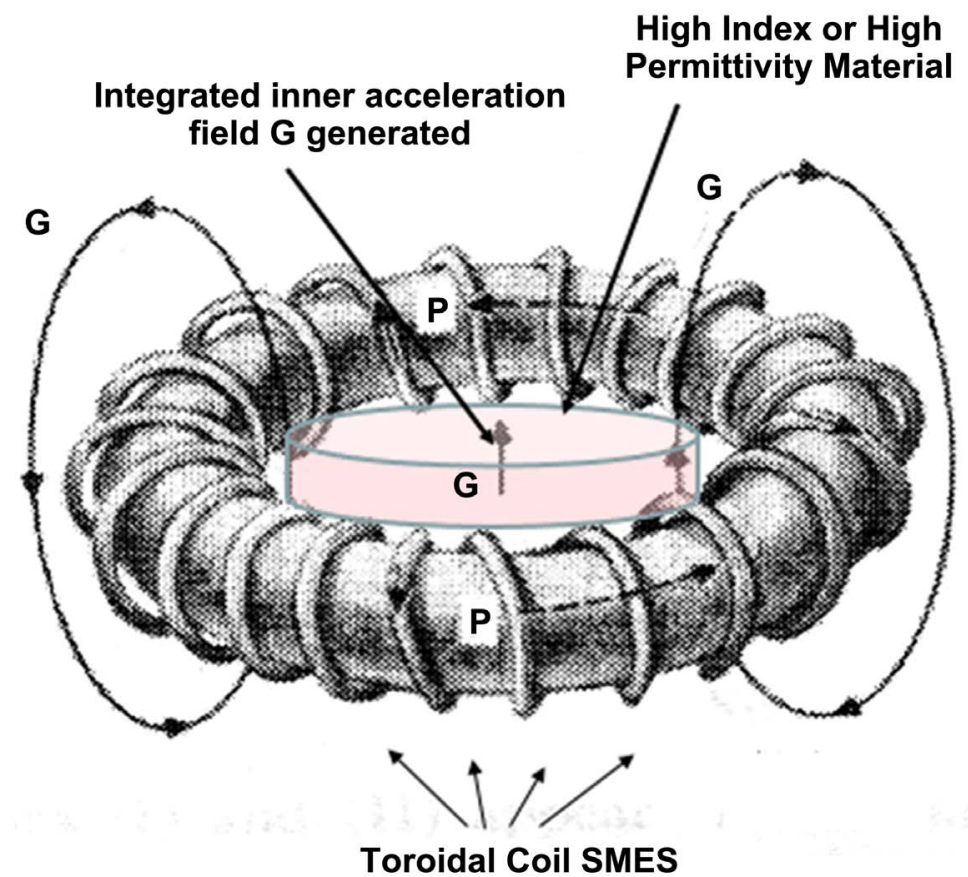

Figure 1. Gravitational force generation coil from Forward 1962 [1] with an inspiralling mass current, with a vector potential $\mathrm{P}$, creating gravitomagnetic field $\mathrm{G}$, with high index material in the center to improve coupling. 
where $\eta$ is gravitomagnetic permeability, $\eta=\eta_{o} \eta_{r}$ as described in reference [2], $N$ is number of turns in the toroid coil winding, $\dot{T}$ represents the mass flow, $r$ is the cross sectional radius of the toroid, and $R$ is the overall radius of the toroid.

Mass flow can be generated by means of generating an electrical current by virtue of putting the mass of electrons in motion.

Electron Mass Flow

Single electron mass flow shown in Figure 2 is given by mass momentum in Equation (2):

$$
T_{e}=p_{e}=(\Omega \times r) m_{e}
$$

where $\Omega=$ angular rate, angular velocity is $v=\Omega \times r$ in the classical case [6].

Change in mass flow for the single electron flow shown in Figure 2 is given by Equation (3):

$$
\dot{T}_{e}=\dot{p}_{e}=a \cdot m_{e}=(\Omega \times v) m_{e}
$$

This is equivalent to centripetal force shown in Equation (4):

$$
\dot{T}_{e}=F_{e}=\frac{m_{e} v^{2}}{r}=m_{e} a_{e}=m_{e}\left(\omega^{2} r\right)
$$

where $\omega$ is the angular rate, given in Equation (5):

$$
\omega=\frac{2 \pi}{t_{p}}=\frac{\mathrm{d} \theta}{\mathrm{d} t}
$$

\section{Current in Idealized SMES}

We now attempt to estimate the possible inspiralling current flux enabled by the emerging SMES technology as it relates to the core geometry constraints described in Figure 3, a toroid with torus geometry. We start with the assumptions needed to calculate the number of turns $N$.

From Equation (1) the torus assumptions made in Figure 3 can be factored into Equation (6) as follows:

$$
G_{f}=\left(\frac{\eta_{o} \eta_{r}}{4 \pi}\right) S\left(\frac{N \dot{T r}^{2}}{R^{2}}\right)
$$

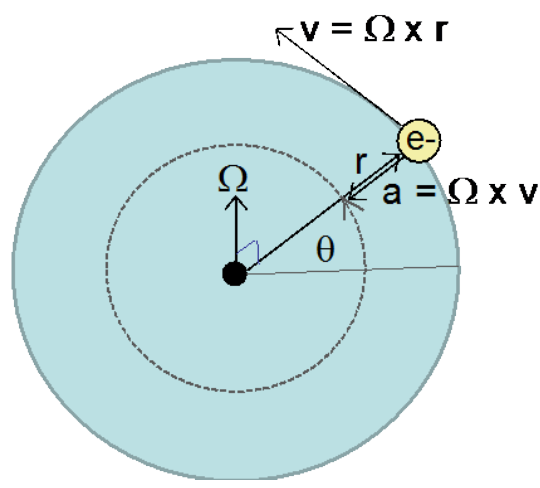

Figure 2. Electron orbit around one loop of SMES toroid. 


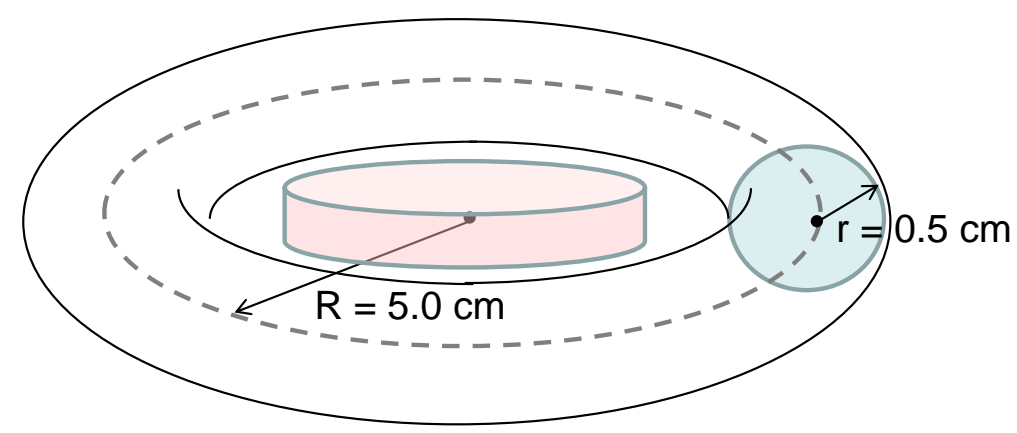

Figure 3. Toroid with a torus shaped core geometry, including high index scaling matter in center.

where:

$G_{f}=$ gravitomagnetic force;

$\eta_{o}=$ absolute gravitomagnetic permeability;

$\eta_{r}=$ relative gravitomagnetic permeability;

$N=$ number of turns in the coil of the torus;

$S=$ Sarfatti scaling factor;

$\dot{T}=$ change in mass, or mass flow;

$r=$ cross section radius of torus;

$R=$ centerline radius of torus.

Note that Equation (6) differs from Forward's original Equation (1) formulation in that it includes the scaling factor $S$ which accounts for the scaling of gravitational coupling due to high index matter or metamaterials, which was first expressed by Sarfatti as an additional zero rank tensor in Einstein's Field Equations [4] [7]:

$$
R_{u v}-\frac{1}{2} R g_{u v}=\left(\frac{8 \pi G}{c^{4}}\right) S T_{u v}
$$

where:

$R_{u v}=$ Ricci curvature tensor;

$R g_{u v}=$ Ricci scalar curvature;

$G=$ gravitational constant;

$c=$ speed of light;

$S=$ Sarfatti scaling tensor;

$T_{u v}=$ Stress Energy Tensor.

For the purposes of describing an idealized case with a realistic geometry we develop a description of a device bounded by a $10 \mathrm{~cm}$ toroid centerline diameter, shown in Figure 3 and Figure 4, with a cross-sectional diameter of $1 \mathrm{~cm}$. Furthermore 16 sectors are defined as shown in Figure 4.

We further add additional assumptions regarding to what extent conductors are wrapped around the toroid shaped device to determine constraints on the number of conductive loops that can be accommodated using the technology described in [2]. As shown in Figure 5 via cross section we assume here a conductor winding depth of $0.1 \mathrm{~cm}$. 


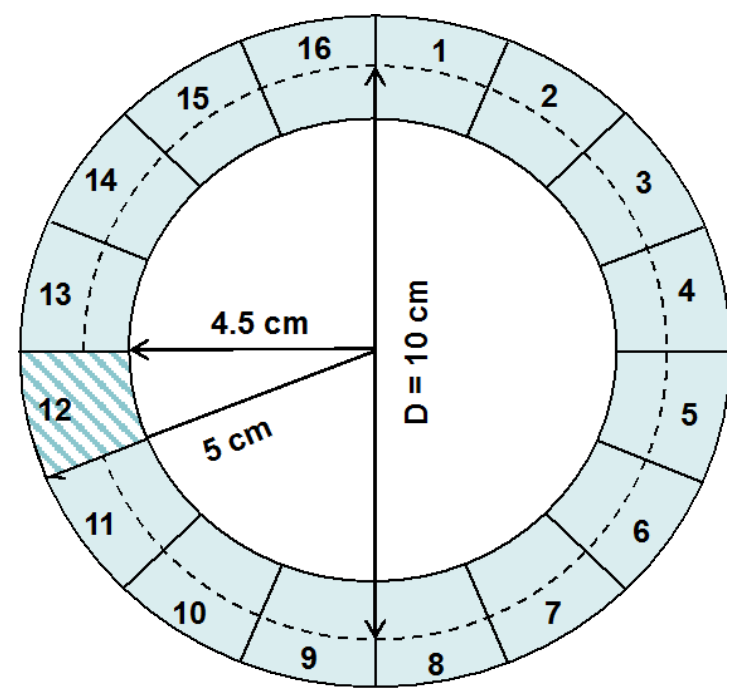

Figure 4. Torus section definition.

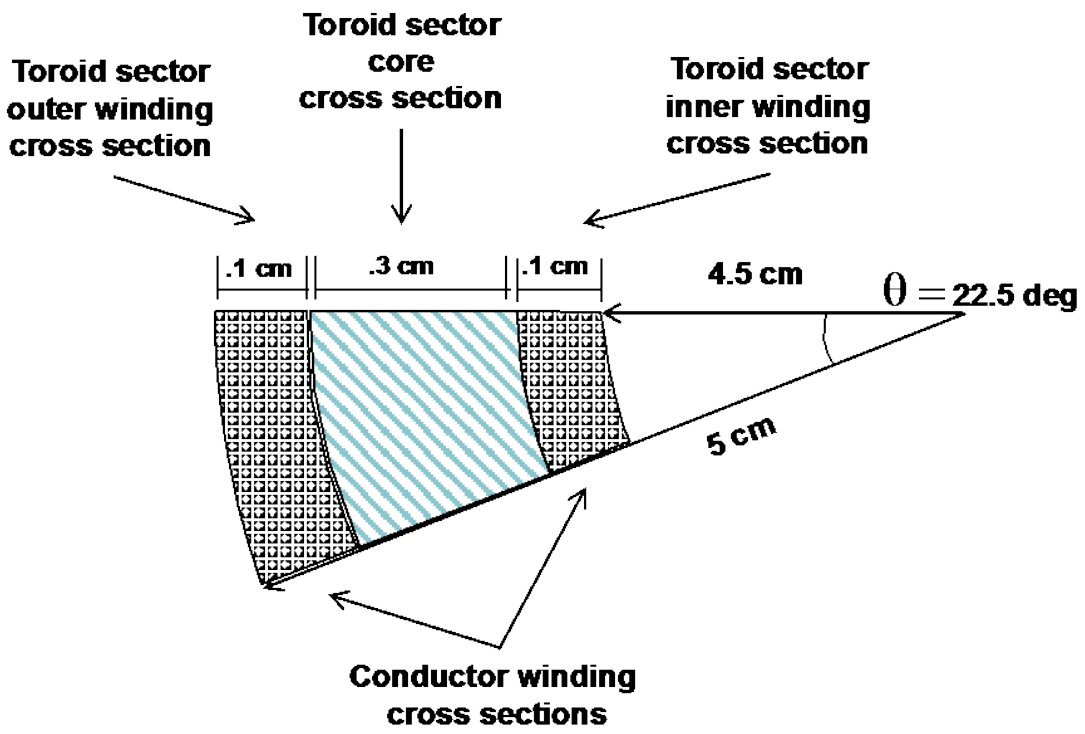

Figure 5. Conductor cross section in each sector.

The segmented share of the inside of cross section $C_{s}$ is $1 / 16^{\text {th }}$ of the overall inner circumference as given in Equation (8) as follows:

$$
C_{s}=\frac{2 \pi r_{i}}{16}=1.77 \mathrm{~cm}
$$

with a depth $D$ the minimum inner loop cross sectional area can be described in Equation (9) as follows:

$$
A_{\text {sec }}=D \cdot C_{s}=0.1 \mathrm{~cm} \times 1.77 \mathrm{~cm}=0.177 \mathrm{~cm}^{2}
$$

This area is shown packed with conductors in Figure 6. Windings are depicted as packed in depth and along sector circumference.

Assuming each nanowire conductor has a diameter $d_{c}$ of $100 \mu \mathrm{m}$, then the cross-sectional area of each conductor will be given by Equation (10) as follows: 


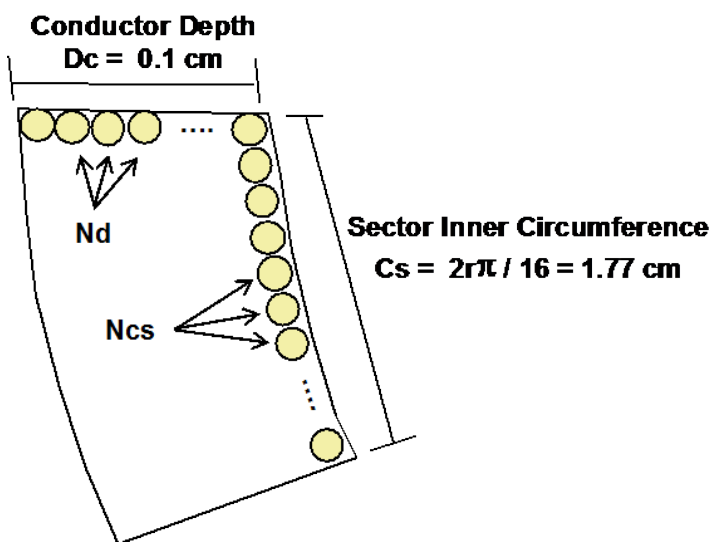

Figure 6. Conductor packing in each sector.

$$
A_{c}=\pi r_{c}^{2}=7.854 \times 10^{-9} \mathrm{~m}^{2}
$$

For packing nanowire conductors in a cross-sectional area described in Figure 6, assume as a worst case rectangular area described by the shortest edges such that a number of conductors in depth, $N_{d}$, may be packed in one dimension, with the number of conductors, $N_{c s}$, packed in the other dimension. These packing counts may be calculated in Equation (11a) and Equation (11b) as follows:

$$
\begin{gathered}
N_{d}=D_{c} / d_{c}=\frac{0.1 \mathrm{~cm}}{100 \mu \mathrm{m}}=10 \\
N_{c s}=C_{s} / d_{c}=\frac{1.77 \mathrm{~cm}}{100 \mu \mathrm{m}}=177
\end{gathered}
$$

The total number of windings by sector will therefore be the product of $N_{d}$ and $N_{c s}$ :

$$
N_{\text {sec }}=N_{d} \cdot N_{c s}=10 \times 177=1770
$$

And with 16 sectors the total number of windings for the entire toroid will be:

$$
N=16 N_{c s}=28320
$$

what is $\dot{T}$ with the forgoing assumptions?

In this idealized case electrons circulate about a coil of circumference $c_{r}$, or slightly larger, as described by Equation (14):

$$
c_{r}=2 \pi r=3.14 \mathrm{~cm}
$$

\section{Electron motion}

Assume further a supply voltage of $16 \mathrm{KV}$, resulting in $16 \mathrm{KeV}$ of kinetic energy for each electron, which corresponds with the upper limit of a non-relativistic case, where $v=0.25 c$, so that $\gamma=1.06-1.0$.

Then from Equation (4) for non-relativistic circular motion, the vector change in DC current flow is:

$$
\dot{T}_{e}=\frac{m_{e} v^{2}}{r}
$$


which for a single electron has the following values:

$m_{e}=$ mass of the electron $=9.11 \times 10^{-31} \mathrm{Kg}$;

$V=$ velocity of the electron $=0.25 c=0.75 \times 10^{8} \mathrm{~m} / \mathrm{s}$;

$r=0.5 \mathrm{~cm}$ for the assumed geometry.

And where the angular acceleration of the electron is:

$$
a_{e}=\frac{v^{2}}{r}=1.125 \times 10^{18} \mathrm{~m} / \mathrm{s}^{2}
$$

Thus, change in mass flow represents centripetal acceleration in the case of circular motion:

$$
\dot{T}_{e}=m_{e} \cdot a_{e}=10.25 \times 10^{-13} \mathrm{~N}
$$

Equation (17) corresponds to the change in mass flow for one electron in one loop of coil. Total mass flow change is therefore the mass flow change per electron times the number of electrons:

$$
\dot{T}=\dot{T}_{e} \cdot N_{e}
$$

Electron current

What is the number of electrons $N_{e}$ in one loop in motion (part of the mass flow) at a given time for an assumed velocity of $v=0.25 c$ ? $N_{e}$ in one loop can be described by the current $I$ times the period of a single loop circulation $\Delta t$.

$$
N_{e}=I \cdot \Delta t
$$

where the period of an orbit can be described by:

$$
\Delta t=\frac{c_{r}}{v}=\frac{2 \pi r}{v}=0.4189 \mathrm{~ns}
$$

what is the possible current inside the idealized device for the case where the entire winding is in series? We make the assumption about max current to stay below critical current density of $250 \mathrm{MA} / \mathrm{m}^{2}$ as described in [2] [3].

Current is limited by the maximum permissible current density and the cross section of the conductor $t$.

$$
I=J \cdot A_{c}
$$

where $J$ is material dependent. For the nanowire assumed in Ref. [2], $J=250$ $\mathrm{MA} / \mathrm{m}^{2}$. Cross sectional area $A_{c}=7.854 \times 10^{-9} \mathrm{~m}^{2}$ as given in Equation (10). Therefore, maximum current for this conductor diameter is $I=1.96$ Amps.

Expanding on Equation (21) the number of electrons $N_{e}$ in circulation in one loop may be calculated by noting that there are $6.2415 \times 10^{18}$ electrons per Coulomb:

$$
N_{e}=\left(\frac{\text { electrons }}{\text { Coulomb }}\right) I\left(\frac{\text { Coulombs }}{\mathrm{sec}}\right) \cdot \Delta t=5.12 \times 10^{9} \text { electrons }
$$

\section{Forces in Idealized SMES}

Expressing Equation (18) as force per electron times the number of electrons in motion in one loop: 


$$
\dot{T}=\dot{T}_{e}\left(\frac{\text { Newtons }}{\text { electron }}\right) \cdot N_{e}(\text { \#electrons })=5.25 \mathrm{mN}
$$

Thus, each loop experiences about $5.25 \mathrm{mN}$ of integrated centripetal force $(\dot{T})$ due to the electrons in circulation within.

We now describe the scale factor to couple this force to the gravitomagnetic effect.

Revisiting Equation (6) which describes the overall linear force developed at the center of the toroidal coil, total gravitomagnetically developed force will be:

$$
G_{f}=\left(\eta_{o} \eta_{r}\right)(S)\left(\frac{N \dot{T}^{2}}{4 \pi R^{2}}\right)=\left(\eta_{o} \eta_{r}\right)(S)(0.118 \mathrm{~N})
$$

where known variables have been grouped on the right and unknown variables have beencollected on the left. This raises the question what are the correct values for $\eta_{o}$ and $\eta_{r}$ ?

Predicted force using gravitational potential scaling

If $\eta_{o}$ goes as $G / 2 c$ as does gravitomagnetic potential (Ref. [8], Equation (1.5)), then:

$$
\eta_{o}=-\frac{G}{2 c}=1.11 \times 10^{-19}
$$

In this case $G_{f}=\left(\eta_{r}\right) 1.31 \times 10^{-20}$. Values of $\eta_{r}$ are experimentally unknown at this time. However if values of $\eta_{r}$ track values of $\varepsilon_{r}^{2}$ then values as high as $\eta_{r}=$ $10^{16}$ may be possible, yielding $G_{f}=0.131 \mathrm{mN}$. For a test mass of $1 \mathrm{Kg}$ this is equivalent to an easily measurable 13.4 micro-g's. This is for a scaling factor of $G / 2 c$. If the scaling factor is $G / c^{2}$ then the effect drops to an unmeasurably low level of $4.47 \times 10^{-14} \mathrm{~g}$ 's, even for $\eta_{r}=10^{16}$.

\section{Predicted force using gravitational field scaling}

If the scaling factor is $G / c^{3}$ or $G / c^{4}$ then the effect is negligible for $\eta_{r}=10^{16}$, and a much higher index material will need to be found to improve coupling, or other means must be found for improving either the $S$ tensor and/or gravitomagnetic permeability $\eta_{r}$.

Consider the case where $\eta_{o}$ goes as $8 \pi G / c^{4}$ as does the coupling constant in Einstein's Field Equations, then:

$$
\eta_{o}=\frac{8 \pi G}{c^{4}}=2.07 \times 10^{-43}
$$

which gives the following expression for total gravitational force developed:

$$
G_{f}=\left(\eta_{o} \eta_{r}\right)(S)(0.118 \mathrm{~N})=\left(\eta_{r}\right)(S) 2.44 \times 10^{-44} \mathrm{~N}
$$

A value of $\eta_{r}=10^{16}$ gives $G_{f}=(S) 4.43 \times 10^{-23}$ Newtons, which requires a scalar value of $S$ of at least an additional $10^{16}$ to obtain a measurable value for $G_{f}$. Per [4] for an isotropic material $S$ can be written as:

$$
S=\frac{1}{2}\left(\epsilon^{2}+\frac{1}{\mu^{2}}\right)=\frac{1}{2}\left(n^{4}+1\right) / \mu^{2}
$$


Therefore either a permittivity of $\varepsilon=10^{8}$ or an index of $n=10^{4}$ with $\mu=1$ would be sufficient to provide the necessary 16 orders of magnitude in coupling improvement. However care should be taken that effects captured by the $S$ factor are not duplicative of those accounted for in the value of $\eta_{r}$.

Per [5] super dielectrics can provide up to $\mathcal{E}=10^{9}$ which, if we simultaneously had $\eta_{r}=10^{16}$ via some unrelated phenomena, would improve the overall generated gravitational force to a marginally measurable level:

$$
G_{f}=\left(\eta_{r}\right)(S) 2.44 \times 10^{-44}=0.244 \mathrm{nN}
$$

Alternatively metamaterials could also be investigated to establish whether non-isotropic materials may create a more advantageous gravitational coupling improvement.

Lenz's Law Implications

Is there an equivalent of Lenz's Law for gravitomagnetics? Consider a hypothetical case where a Forward toroid that is capable of supporting sufficient current and coupling to develop $1 \mathrm{G}$ of acceleration. As a thought experiment imagine disconnecting the power supply and shorting the toroidal coil to itself, such that it is one continuous closed loop of nanowire, and equip a flying craft with such a coil.

At the surface of the Earth such a properly oriented toroidal coil would be immersed in a $1 \mathrm{~g}$ gravitational field. Should not such a field generate a counter-current in a toroidal coil capable of supporting a current of that level, essentially providing a self-powered counter field? If so, a craft so equipped would be able to hover using a counter-field equal but opposite to the Earth's gravitational field without need of additional power sources.

Similarly, additional closed loop toroidal coils, properly oriented, could also be used to "current charge," collecting energy from a static gravitational field, potentially providing power that could be directed to other propulsive or non-propulsive functions of such a hypothetical craft.

\section{Conclusion}

An argument is made for using high permittivity materials in the core or donut hole and/or nanowire insulators of an SMES toroid to improve gravitomagnetic coupling for the creation of an acceleration field, possibly of measurable amplitude. Improved coupling will be beneficial for shrinking device scale and complexity and overcoming the very weak coupling between mass flow and gravitomagnetic spacetime curvature.

\section{Acknowledgements}

The authors wish to acknowledge Jack Sarfatti for helpful correspondence on his insights into altering Einstein's Field Equations in the presence of matter and/or metamaterials. The financial support of Seculine Consulting is gratefully acknowledged. 


\section{Conflicts of Interest}

The author declares no conflicts of interest regarding the publication of this paper.

\section{References}

[1] Forward, R. (1962) Guidelines to Antigravity. Proceedings of the Gravity Research Foundation, New Boston.

[2] Stephenson, G., Rieken, W. and Bhargava, A. (2019) Extended Cases of Laboratory Generated Gravitomagnetic Field Measurement Devices. Journal of High Energy Physics, Gravitation and Cosmology, 5, 375-394. https://doi.org/10.4236/jhepgc.2019.52021

[3] Rieken, W., Bhargava, A., et al. (2018) $\mathrm{YBa}_{2} \mathrm{Cu}_{3} \mathrm{O}_{\mathrm{x}}$ Superconducting NanoRods. Japanese Journal of Applied Physics, 57, 023101. https://doi.org/10.7567/JJAP.57.023101

[4] Stephenson, G. (2020) 2000-2020 Summary of Gravitational Work. APEC Proceedings, Blaine, 12 December 2020.

[5] Fromille, S. and Phillips, J. (2014) Super Dielectric Materials. Materials, 7, 8197-8212. https://doi.org/10.3390/ma7128197

[6] Knudsen, J.M. and Hjorth, P.G. (2000) Elements of Newtonian Mechanics: Including Nonlinear Dynamics. 3rd Edition, Springer, Berlin, 96.

[7] Sarfatti, J. (2020) Explaining US Navy Close Encounters with Tic Tac UAV Metric Engineering. Proceedings of the Estes Park Advanced Propulsion Workshop, Space Studies Institute, North Hollywood, October 2020.

[8] Mashhoon, B. (2008) Gravitoelectromagnetism: A Brief Review. arXiv:gr-qc/0311030v2

\section{Acronyms}

HTSC: High temperature superconductor

SC: Superconductor

SDM: Super dielectric material

SMES: Superconducting magnetic energy storage 